\title{
Elaborating a Decentralized Market Information System
}

\author{
René Brunner and Felix Freitag \\ Computer Architecture Department, Polytechnic University of Catalonia, Spain \\ \{rbrunner, felix\}@ac.upc.edu
}

\begin{abstract}
A Decentralized Market Information System (DMIS) that aggregates and provides information about markets is an important component for achieving markets in Grid and Peer-to-Peer systems. The proposed work is the development of a framework for the DMIS, which fulfils the economic provision within the main technical requirements like scalability towards nodes and data attributes and robustness against failures. The proposed work also allows obtaining results concerning the trade-off between economic benefits and technical costs. Introducing dynamic adaptive processes promises improvements in efficiency with regards to distributed queries and routing structures. This research proposal presents and discusses the research questions and challenges, the current knowledge and the research methodology proposed for the development of the DMIS framework.
\end{abstract}

Key words: Distributed Information System, Monitoring System, Information Aggregation, Publish-Subscribe, Grid Market

\section{Introduction}

With pervasive use of computers and evolving high-speed networks Peer-to-Peer (P2P) systems and Grid systems have emerged as two major solutions for sharing computing resources over the Internet. The assignment of these resources has more recently been proposed to be supported by market models based on bargaining or auctions, like they are pursued in on-going Grid projects CATNETS [1] SORMA [2] and Grid4All [3]. The need for further research in Grid markets is confirmed through the position of the European Union expressed in [4].

This proposal investigates the development of a Decentralized Market Information System (DMIS). This framework aims at providing efficiently economic information about Grid markets to trader agents. To work with different strategies, agents trading in these markets need to acquire some global information in these strictly decentralized large-scale market places. The exact value for accessible information in auction or bargaining markets is still discussed in the economic literature [5]. However, it shows the importance of providing information.

With the Decentralized Market Information System (DMIS), traders in Grid markets can obtain important information needed for the well-functioning of markets. From a technical point of view, details will be observable by choosing 
and comparing with internal components like aggregation models and publishsubscribe systems. An evaluation will provide results on a possible and effective approach that primarily fulfils economic information. Afterwards, with the existing prototype, better and more efficient approaches are planned to obtain with dynamic adaption of internal process structures.

This thesis proposal is organized as follows: Section 2 describes the research questions which the DMIS addresses. Then in Section 3 the research problems for the development of the DMIS framework are explained, which in a technical sense are primary related to the decentralization. The related work is listed in Section 4 , showing differences to the proposed work. Afterwards, some preliminary work done is presented. The section on research methodology shows which steps will be carried out to achieve the research goals. It is followed by a list of the expected contributions. The last section summaries the differences as well as new and better solutions of the planned work.

\section{Research Question}

The planned DMIS framework has to deal with several constraints both from distributed environments, such as scalability and robustness constraints, and from information systems, like privacy, time-sensitivity and historical data availability. That leads to the first open question about the feasibility and integration of the required functionalities and models into one framework. This means not merging directly components since the influence between efficient routing combination of models, the ease of development and flexibility needs to be evaluated. The result to this research question is expected to be given by a well designed architecture, defining the main components and their dependencies.

The research in this thesis should answer the following research question: Is the proposed framework flexibly and reusable in different Grid applications? The result to this question should be answered by validating the DMIS framework with an implementation for market-based Grid projects like Sorma and Grid4All.

A third research question concerns the feasibility of a strictly layered architecture. The DMIS need to interact with two interfaces. The first one is the communication infrastructure provided by a Distributed Hash Table (DHT) implementation. The second one is with a Grid middleware which will consider the DMIS as a service. The results of this question will be determined by the achieved capability of the architecture and implemented prototype to work with different implementations of a required functionality.

How can the information retrieval be optimized, needs to be answered. The information type does not stay static during the application, hence each request requires a different structure or algorithm. Also the storing and retrieval of a large-scale information can be changing meanwhile the application is running. As the prediction of all possible requests would be cost intensive, a dynamic solution during the runtime of the application is required. To ensure an efficient routing with less effort on deploying the application, dynamic adaptation during runtime of the DMIS needs to be investigated. 
Another questions we aim to resolve concerns economic aspects. Is it possible to obtain better price margins once information about the market is available? In central auctions, trading agents with a simple negotiation intelligence need at least some basic information about the market. But in decentralized auctions or bargaining the availability of these values can often not be ensured. The main question is how the economic benefit in form of competitive advantage can be optimized by using the information system.

A challenge is to determine the trade-off between the technical efficiency and the economic profit. In a wide-deployed distributed marketplace it is impossible or cost intensive to guarantee an absolutely exact information about a current state of the market or its participants. This can be the case within complex queries ( e.g., SQL joins ) or by changing values during executing the request. Accepting a slight inaccuracy in information quality could exponentially reduce the cost of technical performance like fewer routing messages and shorter response times.

\section{Problem Formulation}

Trader agents which solve either simple or sophisticated problems need at least some information about the market or about offers from other traders. This supposes no problem in a central organized auction because an auctioneer with an overall knowledge can transmit information to the trader agents. But in decentralized auctions or bargainings, other strategies need to be applied to ensure scalability of information dissemination. Examples of such strategies are dividing auctions in subgroups [6] or lose approaches like Catalactic [7] bargainings. In such a context the overall knowledge gets lost and no accurate information is available (e.g. about the price of all offers in the market).

The decentralization of the market information system faces scalability problems with regards to the number of traders and a high volume of information. Besides the increasing number of participants in the market, especially the requested and released information increase strongly. For example every shouted price shout within a negotiation process has to be published, needs to be received and can be requested by other traders. Furthermore, providing historical data like price charts of the last months, is an expensive task in resource consumption. This requires a high capacity and an equal distribution of the network load to each participant. Moreover, the aggregation of different and complex data may cause higher traffic of messages.

In distributed systems the robustness against a high level of churn (by faults or when participants leave the market) is a common problem. Therefore, the proposed framework needs to be robust against such dynamics in the market. All internal services like providing time-sensitive data, aggregated information or persist and retrieval of historical data have to consider this aspect. Furthermore, a proposed privacy model needs a high level on security, which imposes a robust system. 
Complex information structures causes problems for the retrieving of data from users organized in a DHT. Because all nodes in an aggregation tree needs to be queried, complex queries demand more iterations to obtain an exact result of a distributed value. An alternative is to use approximated queries which only execute one query process but return an inaccurate value. The problem is to find a trade-off between the cost of messages for an exact value and the economic or technical advantage of accurate data. Furthermore, it is almost impossible to return a $100 \%$ exact value in an application with several thousand users. A query needs, in this case, several sequential messages which may not consider changes occurring in meantime. When the retrieved value is returned, the value in the system might has changed.

The architecture of the DMIS builds on various tools which need to be integrated into one framework. Each tool has until now an optimal structure considering an isolated design, but not when they are integrated. For example routing efficiency is different when the persistence of data is pursued with other algorithms than the publish-subscribe or aggregation. Moreover, in an integrated form, these components have to take into account external application interfaces. Each interface needs to be sufficiently flexible to adapt to other applications or frameworks e.g. approaches implementing different DHT algorithms like Pastry [8] or Kademlia 9].

\section{State of the Art}

The planned information system applied to distributed Grid markets is related to academic and commercial monitoring systems described in the survey of [10]. Most of them either follow a centralized approach or use a decentralized approach with a client-server model. As they are designed for Grid systems they focus on technical aspects like the observation of the resource occupation instead of the monitoring of economic values and application layer behavior.

The proposed thesis follows a similar approach like existing peer-to-peer (P2P) aggregation frameworks reached in Astrolabe [1] based on an unstructured P2P overlay. The projects Willow [12, DASIS [13] and SOMO [14] have a single hierarchical tree architecture, that aggregates all attributes along one tree. Consequentially, the root node as well as the hierarchical higher nodes are a point of failure and bears an over proportional network load. Cone [15] has a multiple tree aggregation, where each attribute has its own tree but requires a total order on the attribute values. DSMIS [16] is a hierarchical multiple tree aggregation framework which is based on a DHT overlay and the closest work to the proposed research. The application obtains a high flexibility by deciding the propagation level of the aggregation.

Recent research in P2P-based publish-subscribe models can be categorized into three subscription models: topic-based, content-based and type-based. Topicbased systems like SCRIBE 17] exchange information through a set of predefined subjects called topics. Although these are easy to integrate on top of a DHT, they are not flexible to changes after the creation of a topic. In contrary 
content-based systems like Gryphon [18, Siena [19, Meghdoot 20] and Ferry 21] are more flexible as subscriptions are related to specific information content. Therefore each combination of information items can actually be seen as a single dynamic logical channel. Type-based systems like Hermes [22] are a combination of the previous two approaches; they have the advantage of type-safety that allows the publishers to extend the types of events without forcing the subscribers to update their subscription. Ferry contains more relevant properties like aggregation guaranties, range prediction and persistence. However it is not filter-based which is expected to be an import feature for the DMIS.

Adaptations before deploying applications in distributed software systems result to a high complexity and to be cost intensive. One solution to avoid the prediction of all possible use cases presented in 23], is an automatic adaptation during the application execution. This adaption can be based on dynamic and coordinated actions that adjust an existing structure and the behavior of the system, without an interruption of service.

Some research work about decentralized databases deals with complex queries executed in distributed systems. They consider SQL-like queries [24], which are related to queries on aggregation systems. Therefore the querying principle can be used for the decentralized information recovery. However, its focus differ in providing time-sensitive data.

As the DMIS is proposed for markets, it has a related work in the economic research. A survey from Bergemann [5] discuss the retrieval and aggregation. Some literature profound the theoretical analysis of the influence of information to markets 25. Also works about required information for economic markets are evaluated. However, the above mentioned literature is either simple or focus on theoretical analysis.

\section{Preliminary Work}

Towards achieving the DMIS architecture, we have already characterized and grouped economic requirements in different categories. Furthermore, a new privacy model for market information has been sketched. The defined requirements for the DMIS need specific technical approaches to achieve its implementation. Technical ideas contains for example to introduce an intermedium layer containing mathematical abstractions; this layer maps the requirements into an approximation and subsequently to the applied requests. An example for such an abstraction is the first-order Taylor series approximation instead of disclosing the exact value, which is probably very cost intensive with less value added. For a better realization, filters including these methods will be included.

Figure 1 presents a 3 -tier architecture for the proposed DMIS. It consists of four core services: aggregation, publish-subscribe, archiving or communication built on top of one DHT, which will be Pastry or Kademlia based. The aggregation component is necessary to obtain information in a scalable distributed environment. The publish-subscribe component has to handle and provide timesensitive data. Persistence of the data is handled by the archive component. 
Some privacy constrains require an autonomous communication model for message exchanges. The needed components have to interface with each other as well to connect with the three external interfaces, which are the DHT, the user interface and a Grid market middleware.

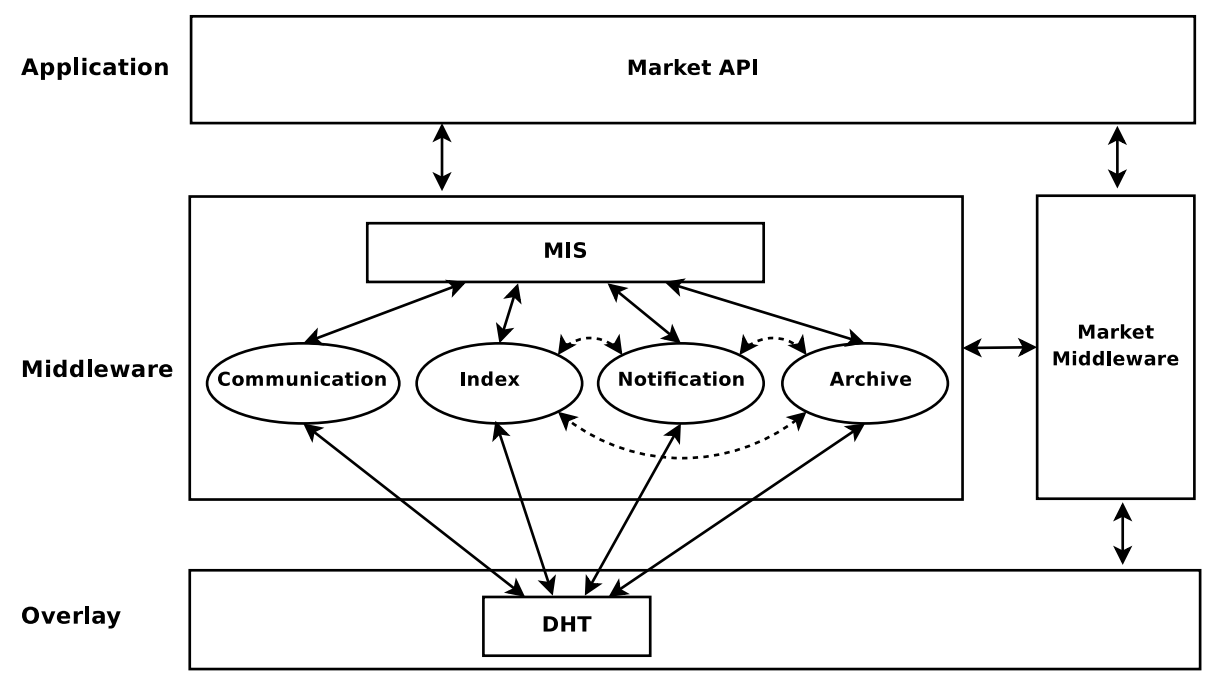

Fig. 1. High-level overview of the DMIS.

\section{Research Methodology}

The proposed research project follows a methodology based on empirical models and quantitative evaluation. The models are obtained by simulations or as results from developed prototypes. The DMIS prototype will demonstrate the feasibility of the framework and the integration of its different components. The behavior will be characterized by stress tests. A benchmark analysis is considered to determine the result without and with optimization of the framework's algorithms.

At the beginning of the project, an agent simulator like Repast [26] or JASA 27. will be used to indicate the economic benefit of information in on-line auctions and bargaining. For this quantitative analysis, several thousands agents will simulate an auction. By providing information about the market to only some agents, the benefit to these agents will be measured. Even a small advantage will be significant as markets can contain high trading volumes. Comparing simulations with information and without information will show the influence, concerning the obtaining of the equilibrium price. After completing the economic analysis, the focus will move to the technical aspects. 
The second step will be the development of a DMIS prototype and the evaluation of its components. Therefore, a detailed survey and taxonomy about P2P-based publish-subscribe models and aggregation model needs to be carried out. Afterwards, the differences between both frameworks will be evaluated. This includes the development of a representative publish-subscribe framework and a representative aggregation framework to compare the efficiency and advantages of both approaches. The most suitable framework builds the base for the DMIS prototype, which will be extended by the other components.

The prototype will be exposed to several tests to show the behavior towards scalability and robustness. An exposure to a stress test will analysis the components under extreme cases. The number of trading agents and the amount of information will be tested to a strong variation. Also complex queries based on SQL allows a wide-range an testing possibilities. Moreover, failures will be simulated by stopping a different number of agents. The framework will be approved when it will be able to resist against those cases and guarantees a successful providing of information.

Since the flexibility of the architecture as regards the internal components and external framework is a main requirement, the integrations with other applications must be tested. This integration capability will be validated by using the DMIS into at least two different Grid market middleware provided by the projects like CATNETS and Grid4All. But the flexibility also means the integration with different DHT overlays. A successful integration will be approved when a selective set of information requests and responses will be provided by all combination of external applications. After the finalization of the prototype and analysis, the optimization of internal processes is envisaged.

After the economic simulations and the development of a functioning prototype, the focus will move to the optimizing and evaluating of internal processes. These processes are primarily retrieval and dissemination of data, which needs different structures for an efficient working by varying type of data and of the system state. Therefore a dynamic adaptation mechanism will be designed and analyzed with a P2P simulator to show the distinction between different adaption capabilities: adaptation before deploying and dynamic adaptation during runtime. An efficient adaptation will be confirmed when the network load and sent messages are less than those obtained without adaptation.

\section{Contribution}

This work is planned to make contribution to the research area of distributed monitoring and information systems applied to market mechanisms. Four mayor contributions can be outlined: development of a new and different framework, the economic analysis of a DMIS in Grid markets, finding a trade-off between technical cost and economic benefit, and introducing better dynamic adaptation approaches in DMIS.

- The development of the new framework DMIS promises to solve problems resulting from decentralization. For the economic information requirements 
of trader agents in Grid markets, the DMIS needs to be based on efficient and complex structures. Furthermore, showing the integration into existing Grid market middleware and integrating the system on top of different DHTbased overlays show the flexibility.

- Economic analysis should show the necessity of a DMIS in on-line markets such as Grid markets. Our work expects to illustrate the advantages for trader agents when they are provided with market information.

- Another contribution will be to investigate the trade-off between costs and benefits. The necessity of exact information is evaluated and the advantage of reducing technical costs like lower network traffic while losing accuracy with approximations.

- The last part of the planned work will contribute to new or better approaches for an automatic adaptation of the structure of the individual DMIS components to ensure efficient processes.

\section{Different, New and Better Solutions}

Only a few researches target a distributed aggregation and a publish-subscribe model in one framework: Willow and DSIMS. Willow combines both approaches in one protocol, which has the trade-off of an effective routing and the design complexity by merging the two approaches. A more important difference is that it focuses on the protocol itself and its effective routing algorithms. Our work will go further in several aspects of privacy, complex queries and adaptive structures.

The most related work is the DSIMS that also aggregates information about large-scale network systems. This work focus on the aggregation of information considering scalability behavior, privacy, robustness and flexibility. A difference to our work is that there is no specific focus on time-sensitive data that can explicitly be addressed. Moreover, in our work the privacy model will be extended and adapted to economic information aspects.

Until now no known work investigates on economic aspects of an applied decentralized information or monitoring system. Therefore, required parameters of general on-line markets as well as Grid markets will be characterized within our thesis. They will be analyzed with a developed DMIS.

The economic literature has discussed about the value of information in markets such as auctions. However, these aspects are still open and are rather theoretical. Our work aims to execute simulations and prototype runs to extract the effort of provided information. These values obtained in simulations can be compared with existing theoretical approaches. Afterwards, its realization in a real environment will be shown and compared with an implemented prototype.

Another distinction is the possibility to integrate the DMIS into several Grid market applications. This integration will show the flexibility and the feasibility of integration into an existing market middleware, into a user interface as front end application and into different DHTs substrates. 


\section{Acknowledgments}

This work was supported in part by the European Union under Contract SORMA EU IST-FP6-034286, and the Ministry of Education and Science of Spain under Contract TIN2006-5614-C03-01.

\section{References}

1. CATNETS Project.Annex I description of work. IST-FP6-003769. 2004.

2. SORMA Project. http://www.iw.unikarlsruhe.de/sormang/.2007.

3. Grid4All Project. http://grid4all.elibel.tm.fr/.2007.

4. Next Generation GRIDs Expert Group. Future for european grids: Grids and service oriented knowledge utilities. ftp://ftp.cordis.lu/pub/ist/docs/grids/ngg3_eg_final.pdf. 2006.

5. D. Bergemann and J. Valimaki. Information in Mechanism Design. Proceedings of the 9th World Congress of the Econometric Society, 2006

6. E. Ogston and S. Vassiliadis, A peer-to-peer agent auction. Proceedings of the First International Joint Conference on Autonomous Agents and Multi-Agent Systems (AAMAS 2002)

7. O. Ardaiz, P. Artigas, T. Eymann, F. Freitag, L. Navarro, and M. Reinicke. The Catallaxy Approach for Decentralized Economic-based Allocation in Grid Resource and Service Markets. International Journal of Applied Intelligence. Special Issue on Agent based Grid Computing. Volume 25 , Issue 2 (October 2006) pages: 131 - 145, 2006

8. A. Rowstron and P. Druschel. Pastry: Scalable, distributed object location and routing for large-scale peer-to-peer systems. In Proc. IFIP/ACM Middleware 2001, Heidelberg, Germany, November 2001.

9. P. Maymounkov and D. Mazieres. Kademlia: A Peer-to-peer Information System Based on the XOR Metric. In Proceesings of the IPTPS, March 2002.

10. S. Zanikolas and R. Sakellariou, A taxonomy of grid monitoring systems. Future Generation Computer Systems 21, 1, 163-188. 2005

11. R. van Renesse, K. Birman, and W. Vogels. Astrolabe: A robust and scalable technology for distributed system monitoring, management and data mining. ACM Transactions on Computer Systems, vol. 21, no. 2, May 2003.

12. R. van Renesse and A. Bozdog. Willow: DHT, Aggregation, and Publish/Subscribe in One Protocol. Proc. 3rd Int. Workshop on Peer-To-Peer Systems (IPTPS) 2004.

13. K. Albrecht, R. Arnold, M. Gahwiler, and R. Wattenhofer. Join and Leave in Peerto-Peer Systems: The DASIS approach. Technical report, CS, ETH Zurich, 2003.

14. Z. Zhang, S.-M. Shi, and J. Zhu. SOMO: Self-Organized Metadata Overlay for Resource Management in P2P DHT. In IPTPS, 2003.

15. R. Bhagwan, P. Mahadevan, G. Varghese, and G. M. Voelker. Cone: A Distributed Heap-Based Approach to Resource Selection. Technical Report CS2004-0784, UCSD, 2004.

16. P. Yalagandula and M. Dahlin. A scalable distributed information management system. SIGCOMM '04: Proceedings of the 2004 conference on Applications, technologies, architectures, and protocols for computer communications,pages 379-390, 2004.

17. M. Castro, P. Druschel, A.-M. Kermarrec, and A. Rowstron. SCRIBE: A Largescale and Decentralised Application-level Multicast Infrastructure. IEEE JSAC (Special issue on Network Support for Multicast Communications), 2002. 
18. G. Banavar, T. Chandra, B. Mukherjee, J. Nagarajarao, R.E. Strom, and D.C. Sturman. An Efficient Multicast Protocol for Content-Based Publish-Subscribe Systems. International Conference on Distributed Computing Systems (ICDCS'99).

19. A. Carzaniga, D. S. Rosenblum, and A. L. Wolf. Design and Evaluation of a Wide-Area Event Notification Service. ACM Transactions on Computer Systems, 19(3):332.383, 2001

20. A. Gupta, O. D. Sahin, D. Agrawal, and A. E. Abbadi. Meghdoot: Content-Based Publish/Subscribe over P2P Networks. In Proceedings of ACM/IFIP/USENIX 5th International Middleware Conference. 2004

21. Y. Zhu, and Y. Hu. Ferry: An Architecture for Content-Based Publish/Subscribe Services on P2P Networks. International Conference on Parallel Processing (ICPP'05).

22. P. R. Pietzuch, J. M. Bacon. Hermes: distributed event-based middleware architecture. International Workshop on Distributed Event-Based Systems (DEBS'02).

23. G. Valetto, D. Phung, and G. Kaiser. A Uniform Programming Abstraction for Effecting Autonomic Adaptations onto Software Systems. In Proceedings of the 2nd IEEE International Conference on Autonomic Computing (ICAC 2005), June 2005, Seattle, WA, USA.

24. R. Huebsch, B. Chun, J. M. Hellerstein, B. T. Loo, P. Maniatis, T. Roscoe, S. Shenker, I. Stoica, and A. R. Yumerefendi. The Architecture of PIER: an InternetScale Query Processor. Proceedings of Second Biennial Conference on Innovative Data Systems Research (CIDR 2005), pages 28-43, 2005.

25. M. O. Jackson. Efciency and information aggregation in auctions with costly information. Review of Economic Design, 8, pages 121412003.

26. Collier, N. RePast: An extensible framework for agent simulation. http://repast. sourceforge.net. 2004

27. Steve Phelps. Java Auction Simulator API. http://www.csc.liv.ac.uk/ sphelps/jasa/. 2005 